\title{
Correction to: Behçet's disease in Egypt: a multicenter nationwide study on 1526 adult patients and review of the literature
}

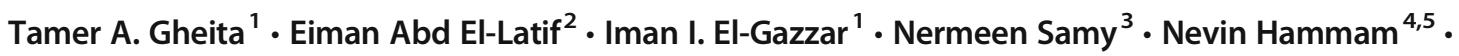
Rasha A. Abdel Noor ${ }^{6}$. Emad El-Shebeiny ${ }^{7}$. Amany R. El-Najjar ${ }^{8}$ • Nahla N. Eesa ${ }^{1}$ - Mohamed N. Salem ${ }^{9}$. Soha E. Ibrahim ${ }^{10}$. Dina F. El-Essawi ${ }^{11}$. Ahmed M. Elsaman ${ }^{12}$ - Hanan M. Fathi ${ }^{13}$. Rehab A. Sallam ${ }^{14}$.

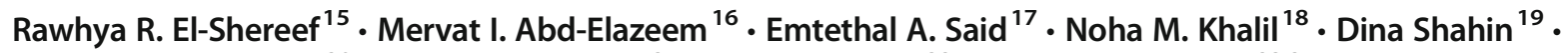
Hanan M. El-Saadany ${ }^{20}$. Marwa S. ElKhalifa ${ }^{21}$ - Samah I. Nasef ${ }^{22}$ - Ahmed M. Abdalla ${ }^{23,24}$. Nermeen Noshy ${ }^{3}$. Rasha M. Fawzy ${ }^{17}$. Ehab Saad ${ }^{25}$. Abdel-Hafeez Moshrif ${ }^{26}$ - Amira T. El-Shanawany ${ }^{27}$ - Yousra H. Abdel-Fattah ${ }^{28}$. Hossam M. Khalil ${ }^{29}$ • Egyptian College of Rheumatology-Behçet's Disease Study Group (ECR-BDSG)

Published online: 13 July 2019

(C) International League of Associations for Rheumatology (ILAR) 2019

\section{Correction to: Clinical Rheumatology https://doi.org/10.1007/s10067-019-04570-w}

The author wishes to correct the record and clarify that in the original version of this article in the Discussion section under "Prevalence over the country governorates" inadvertently presented incorrect data cited in the reference [30]. Originally reading "The prevalence rate in the North of Jordan was estimated as 6.6:100,000 and was considered high in relation to other countries in the universe [30]." this should have read as "The prevalence rate in the North of Jordan was estimated as 660:100.000 and was considered high in relation to other countries in the universe [30]." [bold text used to highlight problem area]

Publisher's note Springer Nature remains neutral with regard to jurisdictional claims in published maps and institutional affiliations.

The online version of the original article can be found at https://doi.org/ 10.1007/s10067-019-04570-w

Tamer A. Gheita

gheitamer@hotmail.com; http://scholar.cu.edu.eg/?q=rheumatalk/

Extended author information available on the last page of the article 


\section{Affiliations}

Tamer A. Gheita ${ }^{1}$ - Eiman Abd El-Latif ${ }^{2}$ - Iman I. El-Gazzar ${ }^{1}$ - Nermeen Samy ${ }^{3} \cdot$ Nevin Hammam $^{4,5}$. Rasha A. Abdel Noor ${ }^{6}$ - Emad El-Shebeiny ${ }^{7}$ - Amany R. El-Najjar ${ }^{8}$ - Nahla N. Eesa ${ }^{1}$ • Mohamed N. Salem ${ }^{9}$. Soha E. Ibrahim ${ }^{10}$. Dina F. El-Essawi ${ }^{11}$. Ahmed M. Elsaman ${ }^{12}$ • Hanan M. Fathi ${ }^{13} \cdot$ Rehab A. Sallam $^{14}$. Rawhya R. El-Shereef ${ }^{15} \cdot$ Mervat I. Abd-Elazeem $^{16} \cdot$ Emtethal A. Said $^{17} \cdot$ Noha M. Khalil $^{18} \cdot$ Dina Shahin $^{19}$. Hanan M. El-Saadany ${ }^{20}$. Marwa S. ElKhalifa ${ }^{21}$ - Samah I. Nasef ${ }^{22} \cdot$ Ahmed M. Abdalla $^{23,24}$ • Nermeen Noshy ${ }^{3}$. Rasha M. Fawzy ${ }^{17}$. Ehab Saad ${ }^{25}$ • Abdel-Hafeez Moshrif ${ }^{26}$ • Amira T. El-Shanawany ${ }^{27}$ • Yousra H. Abdel-Fattah ${ }^{28}$. Hossam M. Khalil ${ }^{29}$

1 Rheumatology Department, Faculty of Medicine, Cairo University, Cairo, Egypt

2 Ophthalmology Department, Faculty of Medicine, Alexandria University, Alexandria, Egypt

3 Internal Medicine Department, Rheumatology Unit, Faculty of Medicine, Ain-Shams University, Cairo, Egypt

4 Rheumatology Department, Faculty of Medicine, Assuit University, Assuit, Egypt

5 Faculty of Medicine and Dentistry, University of Alberta, Edmonton, Canada

6 Internal Medicine Department, Rheumatology Unit, Tanta University, Tanta, Gharbia, Egypt

7 Internal Medicine Department, Rheumatology Unit, Menoufia University, Shebeen El-Kom, Menoufia, Egypt

8 Rheumatology Department, Faculty of Medicine, Zagazig University, Zagazig, Sharkia, Egypt

9 Internal Medicine Department, Rheumatology Unit, Faculty of Medicine, Beni-Suef University, Beni-Suef, Egypt

10 Rheumatology Department, Faculty of Medicine, Ain Shams University, Cairo, Egypt

11 Internal Medicine Department, Rheumatology Unit (NCRRT), Atomic Energy Authority (AEA), Cairo, Egypt

12 Rheumatology Department, Faculty of Medicine, Sohag University, Sohag, Egypt

13 Rheumatology Department, Faculty of Medicine, Fayoum University, Fayoum, Egypt

14 Rheumatology Department, Faculty of Medicine, Mansoura University, Mansoura, Dakahlia, Egypt

15 Rheumatology Department, Faculty of Medicine, Minia University, Minia, Egypt
16 Rheumatology Department, Faculty of Medicine, Beni-Suef University, Beni-Suef, Egypt

17 Rheumatology Department, Faculty of Medicine, Benha University, Benha, Kalubia, Egypt

18 Internal Medicine Department, Rheumatology Unit, Faculty of Medicine, Cairo University, Cairo, Egypt

19 Internal Medicine Department, Rheumatology Unit, Faculty of Medicine, Mansoura University, Mansoura, Dakahlia, Egypt

20 Rheumatology Department, Faculty of Medicine, Tanta University, Tanta, Egypt

21 Internal Medicine Department, Rheumatology Unit, Faculty of Medicine, Alexandria University, Alexandria, Egypt

22 Rheumatology Department, Faculty of Medicine, Suez-Canal University, Ismailia, Egypt

23 Rheumatology Department, Faculty of Medicine, Aswan University, Aswan, Egypt

24 Internal Medicine Department III, Division of Rheumatology, Medical University of Vienna, Vienna, Austria

25 Rheumatology Department, Faculty of Medicine, South Valley University, Qena, Egypt

26 Rheumatology Department, Faculty of Medicine, Al-Azhar University, Assuit, Egypt

27 Rheumatology Department, Faculty of Medicine, Menoufia University, Shebeen El-Kom, Menoufia, Egypt

28 Rheumatology Department, Faculty of Medicine, Alexandria University, Alexandria, Egypt

29 Ophthalmology Department, Faculty of Medicine, Beni-Suef University, Beni-Suef, Egypt 